Running head: Visual search for people

\title{
Visual search for people among people
}

Liuba Papeo ${ }^{1 *}$, Nicolas Goupil ${ }^{1}$, Salvador Soto-Faraco ${ }^{2}$

${ }^{1}$ Institut des Sciences Cognitives-Marc Jeannerod, Unité Mixte de Recherche (UMR) 5229, Centre National de la Recherche Scientifique (CNRS) and Université Claude Bernard Lyon $1 ;{ }^{2}$ Center for Brain and Cognition, Universitat Pompeu Fabra.

*Corresponding author: CNRS, Institut des Sciences Cognitives " Marc Jeannerod », 67 Boulevard Pinel, 69675, Bron, France. Email: liuba.papeo@isc.cnrs.fr

Word count (intros, discussion, and acknowledgments): 1859 


\section{Abstract (143 words)}

Humans can effectively search visual scenes by spatial location, visual feature or whole object. Here, we show that visual search can also benefit from fast appraisal of relations between individuals in human groups. Healthy adults searched for a facing (seemingly interacting) body-dyad among nonfacing dyads, or vice versa. We varied the task parameters to emphasize processing of targets or distractors. Facingdyad targets were more likely to recruit attention than nonfacing-dyad targets (Experiments 1-2-4). Facing-dyad distractors were checked and rejected more efficiently than nonfacing-dyad distractors (Experiment 3). Moreover, search for an individual body was harder when it was embedded in a facing, than a nonfacing dyad (Experiment 5). We propose that fast grouping of interacting bodies in one attentional unit is the mechanism that accounts for efficient processing of dyads within human groups, and for the inefficient access to individual parts within a dyad. 


\section{Introduction (764)}

Recognition of biological entities is vital to human survival. Ancestral motives, lifelong experience and internal motivations are thought to account for the prioritization by human visual attention, of properties and stimuli such as biological motion, eye-gaze, faces and bodies (Birmingham \& Kingstone, 2008; Downing, Bray, Rogers, \& Childs, 2004; New, Cosmides \& Tooby, 2007; Papeo, Wurm, Oosterhof, \& Caramazza, 2017).

We propose that, for humans, no less important than recognition of biological entities is recognition of ongoing social exchanges. Observed interactions between two or more individuals must entail rapid discrimination in order to activate adaptive behaviors such as defense, assistance or cooperation (Quadflieg \& Koldewyn, 2017). Through third-party interactions, individuals can rapidly infer group affiliation, social conventions and conformity (Powell \& Spelke, 2018).

Specific perceptual adaptations might meet the requirement for efficient processing of third-party interactions, allowing, for example, efficient detection (Papeo, Stein \& Soto-Faraco, 2017). Such mechanism could be particularly crucial for parsing crowded scenes (i.e., scenes with multiple preferred stimuli such as faces and bodies), and select and prioritize the portion in which a social exchange is unfolding.

The mechanisms for parsing and selection in cluttered environments have been extensively studied with visual search. Typically, the subject searches for a target among a set of distractors. Search efficiency, the rate at which items are processed, can be affected by task demand, properties of the array (e.g., spatial frequency and predictability of target location), perceptual or semantic properties of target and 
distractors, and the relation between them (Duncan \& Humphreys, 1989). Moreover, given two kinds of stimuli, searching for $A$ among $B s$ can be more efficient than searching for $B$ among $A s$ (Treisman \& Souther, 1985). Such asymmetry may occur because target $A$ is more salient than $\operatorname{target} B$, or distractors $B s$ are easier to check and reject than distractors As. Moreover, depending on the search protocol, one effect or the other can express the asymmetry between two stimuli.

Here, we addressed whether two bodies within a crowd are detected and processed more efficiently if they appear to interact than if they appear unrelated. The asymmetry between searching for a facing dyad among nonfacing dyads and searching for a nonfacing dyad among facing dyads, was tested in visual search tasks, which emphasized the effect of processing either the targets or the distractors. In Experiments 1-2 and 4, different versions of visual search shared one feature: the target always appeared in a subset of central locations. This was done to favor target detection (Carrasco, McLean, Katz \& Frieder, 1998; Neider \& Zelinsky, 2008) and, thus, probe whether recruitment of attention by the facing target was stronger than by the nonfacing target. In Experiment 3, we increased the target location uncertainty to push search through the whole array. Under these circumstances, search times tend to reflect how fast distractors -the majority of items in the arraycan be checked out. In Experiments 1-4, we also varied the set size, in order to measure whether search efficiency (i.e., time/item) changed when searching through facing versus nonfacing distractors, as we specifically predicted for Experiment 3.

Finally, Experiment 5 sought to unravel the mechanism beyond the putative priority 
of facing dyads in search. It assessed whether such advantage is mediated by perceptual grouping, the processing of two items as one attentional unit. We reasoned that, because the face-to-face positioning is a perceptual cue to human interactions, two facing bodies could be processed as a unit while parsing a scene. A similar kind of grouping mechanism has been suggested for (seemingly interacting) objects (Riddoch et al., 2003). Grouping resolves in more efficient processing of the configuration, but involves a cost for individuating parts within it. For example, search for a face is more efficient than searching for a component within, such as the mouth (Suzuki \& Cavanagh, 1995). Since the search mechanism accesses the highest level of representation available, to the detriment of local features, we hypothesized that the advantage of accessing a facing dyad has a cost in terms of access to its local features.

In sum, while research has extensively studied target selection by spatial location, visual features or object identity, the present study provides the first test of whether visual search affords a fast appraisal of relations between people in a crowd. Experiments 1-4 provide twofold information on the role of visual relations between bodies in visual search, and the perceptual advantage of facing over nonfacing dyads. Experiment 5 addresses grouping of multiple bodies in visual search, by targeting the expected cost on accessing a local feature (an individual) of a hierarchically higher representation (a dyad).

\section{Experiment 1 (29)}

Here, we set conditions to test whether, in a crowd, two facing bodies were more likely to recruit attention than the same two bodies facing away from each other. 


\section{Participants}

Eleven healthy adults (10 female, 23 years $\pm 2.48 S D$ ) with normal or corrected-tonormal vision participated as paid volunteers, after giving informed consent. The local ethics committee (Comités de protection des personnes Sud-Est II) approved this and the following experiments. The sample size was decided following previous studies that provided the background knowledge for the current research (Kaiser, Stein, \& Peelen, 2014; Suzuki \& Cavanagh, 1995; Wolf, 2001). Sensitivity power analysis ( $G *$ Power 3.1 ) showed that a sample size of $11(\beta=0.80$, alpha $=0.05)$ had power for a minimal detectable effect size of $\eta_{p}{ }^{2}=0.293$ in the critical interaction between target, orientation of distractors and set size (see below).

\section{Stimuli and apparatus}

Stimuli were search arrays composed of eight or twelve body dyads (see examples in Figure 1). Thirty-three dyads were created using two of ten bodies in different poses in left and right profile, for a total of 20 bodies. Bodies were grey-scale models created with Daz3D (Daz Productions, Salt Lake City, UT) and the MATLAB Image Processing Toolbox (The MathWorks, Natick, MA). All body poses were biomechanically possible and suitable to a social context. By varying the poses in targets and distractors, across trials and across subjects, we could test the processing of spatial relations (facing versus nonfacing) across different instances of those relations, minimizing the impact of low level features of individual stimuli.

Each dyad included one body oriented leftward and one body oriented rightward, which could face toward or away from each other. Distances between two bodies in a dyad were matched across facing and nonfacing dyads. Distance was defined as 
both the distance between the centers of the two bodies (mean facing $_{210}$ pixels \pm 1.47SD; mean nonfacing $_{210}$ pixels $\left.\pm 2.37 S D ; t(64)=0.49, p>0.250\right)$, and the distance between the two closest extremities of the two bodies $\left(\right.$ mean $_{\text {facing }} 62.57$ pixels \pm 13.27SD; mean $_{\text {nonfacing }} 62$ pixels $\left.\pm 13.43 S D ; t(64)=0.17, p>0.250\right)$.

Each search array was divided in two halves with a central fixation-cross. Each half was divided in eight cells (two columns of four cells) with slightly shifted onsets along the vertical and horizontal axis, for a total of 16 cells. Four or six dyads, all facing or all nonfacing, appeared on one side of the array. The other side was the mirror version of the first. The two halves differed only for one cell: on either half, this cell featured a facing dyad (the target), when all other cells featured nonfacing dyads ( $50 \%$ trials), and a nonfacing dyad (the target), when all other cells displayed facing dyads ( $50 \%$ trials). In each array, the distractors could appear in any of the 16 cells, while the target could only appear in one of the eight locations around central fixation, where the greater spatial resolution of vision could favor detection (Carrasco, McLean, Katz \& Frieder, 1998).

For each subject, a unique set of stimuli was created including 400 arrays with a facing-target among nonfacing-distractors (facing-target condition) and 400 arrays with a nonfacing-target among facing-distractors (nonfacing-target condition). Distractors could appear upright or inverted (rotated by $180^{\circ}$ ). Inversion disrupts body representation by disrupting the spatial relations between parts, but leaves unaltered all the low-level visual features (Reed, Stone, Grubb, \& McGoldrick, 2006). The condition with inverted distractors served to control whether search asymmetry 
depended on low-level properties of the distractors and/or a general bias for one dyad or the other, rather than the hypothesized body-to-body relationship.

In each of the two conditions, half of the trials included eight pairs (one target among seven distractors) and the other half, twelve pairs (one target among eleven distractors). In summary, each subject saw 800 unique arrays including a facingtarget among seven or eleven upright or inverted non-facing distractors (100 trials for each condition) and a nonfacing-target among seven or eleven upright or inverted facing-distractors (100 trials per condition). Images were displayed on a 17inch CRT monitor (1024 x 768 pixel resolution, $85-\mathrm{Hz}$ refresh rate), $60 \mathrm{~cm}$ away from the subject's eyes. Individual dyads subtended $\sim 1.5^{\circ}$ of visual angle $\left(\sim 0.6^{\circ}\right.$ for a single body) and were separated by $\sim 3.5^{\circ}$ of visual angle. The array did not exceed $18^{\circ}$ of visual angle. Stimulus presentation and response collection were controlled through the Psychophysics Toolbox extension of MATLAB (Brainard, 1997).

None of the experiments reported in this article was formally preregistered. Request for the materials, as well as the data, can be sent via email to the corresponding author.

\section{Procedure}

Subjects seated on a height-adjustable chair, $60 \mathrm{~cm}$ away from a computer screen, with eyes aligned to the center of the screen. In two separate blocks, subjects were instructed to search for the only facing dyad among nonfacing dyads or vice versa, and report whether the target was on the left or on the right side of the screen. They had to respond by pressing one of two keys (the key " $\mathrm{z}$ " located on the left side or the key "1" located on the right side of a computer keyboard) with the left and right 
index finger, respectively. The key assignment (responding to left-sided targets with the left key/hand and to right-sided targets with the right key/hand) was the same for all subjects to avoid stimulus-response incongruence (i.e., responding to leftsided targets with the right key/hand and to right-sided targets with the left key/hand). This choice had no consequence on the results, as responses to trials with left-sided and right-sided targets were averaged across all experimental conditions. The order of blocks (facing target-nonfacing target or nonfacing target-facing target) was alternated across subjects. Each trial began with a central-fixation cross (200 ms), followed by a blank (700 ms) and then a search array, shown for $800 \mathrm{~ms}$. After the search array disappeared, a blank was shown until the subject's response. The next trial began after 1400 ms. Subjects were invited to take a break every 40 trials, and in the interval between the two blocks. The experiment began with a familiarization block including 16 stimuli, two for each of the eight experimental conditions. The entire experiment lasted $\sim 75$ minutes.

In sum, Experiment 1 included three important features (see Table 1 for a summary). First, to favor detection, the target always appeared in a subset of central locations, where spatial resolution is higher than at greater eccentricities (Carrasco, McLean, Katz, \& Frieder, 1998). In this condition, subjects may implicitly learn to focus on a subset of locations, which can further increase search efficiency (Neider \& Zelinsky; 2008; Wolfe, Alvarez, Rosenholtz, Kuzmova, \& Sherman, 2011). Second, detection was further promoted by short stimulus presentation, which minimized eye movements and covert shifts of attention away from the task-relevant central area (Carrasco et al., 1998; Kwak, Dagenbach, \& Egeth, 1991). Third, distractors were presented upright or inverted. Inversion disrupts body representation by disrupting 
the spatial relations between parts, but leaves unaltered all the low-level visual features (Reed, Stone, Grubb, \& McGoldrick, 2006). The condition with inverted distractors served to control that any search asymmetry found in the condition with upright distractors, depended on the relationship between target and distractors, rather than on low-level properties of the distractors and/or a general bias for one dyad or the other.

\section{Results}

We computed the mean proportion of correct responses (accuracy) and the mean RT for each condition for each subject. All subjects' mean values were within $2.5 S D$ from the group mean; therefore, they were all included in the following analyses. We conducted 2 target type (facing dyad, nonfacing dyad) $\times 2$ orientation of distractors (upright, inverted) $\times 2$ set size (eight, twelve) repeated-measures analyses of variance (ANOVAs) on accuracy values and RTs.

For the accuracy analysis, the results showed a search asymmetry only in the condition with upright distractors: accuracy was higher in search for the facing target among nonfacing distractors than in the opposite condition (Figure 1b). This pattern was confirmed by a significant interaction between target type and distractors' orientation, $F(1,10)=8.43, p=.016, \eta_{p}^{2}=0.457$. A significant interaction between the three factors showed that this interaction was stronger with set size of eight, than with set size of twelve, $F(1,10)=30.86, p<.001, \eta_{p}{ }^{2}=0.755$. Pairwise comparisons showed that the search advantage for the facing target among nonfacing distractors was only significant in the condition with set size eight and upright distractors, $t(10)=4.15, p=0.002$ (all other $p s>0.11$ ). This effect was found 
in nine out of eleven subjects. The overall ANOVA also showed a trend for a main effect of target type, $F(1,10)=4.31, p=.065, \eta_{p}{ }^{2}=0.301$, and significant effects of orientation of distractors, $F(1,10)=38.18, p<.001, \eta_{p}{ }^{2}=0.792$, and set size, $F(1,10)=$ 9.29, $p=0.012, \eta_{p}{ }^{2}=0.481$. Also significant was the interaction between distractors' orientation and set size, $F(1,10)=5.42, p=.042, \eta_{p}{ }^{2}=0.351$. The interaction between target and set size was not significant, $F(1,10)=2.27, p=.163, \eta_{p}{ }^{2}=0.185$. All significant effects and interactions were qualified by the above three-way interaction.

For the RT analysis, we considered values from trials in which subjects provided a correct response and within 2 SD from the individual's mean (79\% of total values). No asymmetry was found. The only significant results were the main effect of distractors' orientation, $F(1,10)=31.68, p<.001, \eta_{p}{ }^{2}=0.760$, the main effect of set size, $F(1,10)=14.26, p=.004, \eta_{p}^{2}=0.588$, and the interaction between the two, showing that the RT difference across different set-size conditions was larger with upright, than inverted distractors, $F(1,10)=10.36, p=.009, \eta_{p}{ }^{2}=0.509$ (Figure 1b). All other effects or interactions were far from significant (effect of target, target $x$ distractors' orientation, and target $\mathrm{x}$ set size: $F s(1,10)<1$, ns; target $\mathrm{x}$ distractors' orientation $\mathrm{x}$ set size, $\left.F(1,10)=1.77, p=.213, \eta_{p}{ }^{2}=0.150\right)$. Given the lack of interaction between target type and set size, the search efficiency reflected by the search slope (time(ms)/item) was comparable in the two target-type conditions.

In summary, Experiment 1 revealed a search asymmetry in a task where the relation between targets and distractors concerned the relative positioning of bodies (facing/nonfacing) in a dyad. The asymmetry was such that subjects were more 
accurate in detecting a facing dyad among nonfacing dyads, than in the opposite condition. This effect was not found when distractors were inverted. With inverted distractors, both targets popped out quite strongly, as shown by high accuracy rates. This rules out the possibility that the asymmetry found with upright distractors was due to low-level properties of the stimuli (which were preserved in the condition with inverted distractors), or to a general bias for facing dyads, independent from the relation between targets and distractors. Moreover, the asymmetry was reliable only with a set size of eight dyads (i.e., 16 bodies); the search through twelve dyads (i.e., 24 bodies) within 800 ms was probably too difficult to appreciate an effect. Indeed, search for the facing target was overall better with a set size of eight than twelve, $t(10)=3.94, p<0.003$

Both RTs and accuracy showed a main effect of set size (the more the distractors, the slower/less accurate the performance). This could reflect a general effect of crowding (or stimulus density), given that the area of the array and the size of the bodies were constant across set sizes. With the increase of spatial density, effects of crowding and lateral interference increase, particularly when items have high visual similarity, like in the current case (Carrasco et al., 1998). However, the effect of set size did not interact with the target type. The absence of this interaction, particularly in RTs, implies that search was equally efficient in the two target-type conditions, but subjects were more likely to detect/recognize correctly the facing, than the nonfacing target. A possibility is that, in the stimulus-presentation short time, the facing target around central fixation could recruit attention more strongly that the nonfacing target. This interpretation is supported by prior findings showing that recognition of facing-body dyads is more efficient than nonfacing-body dyads, using 
a different protocol with brief and spatially certain presentations of the stimuli

(Papeo et al., 2017).

A
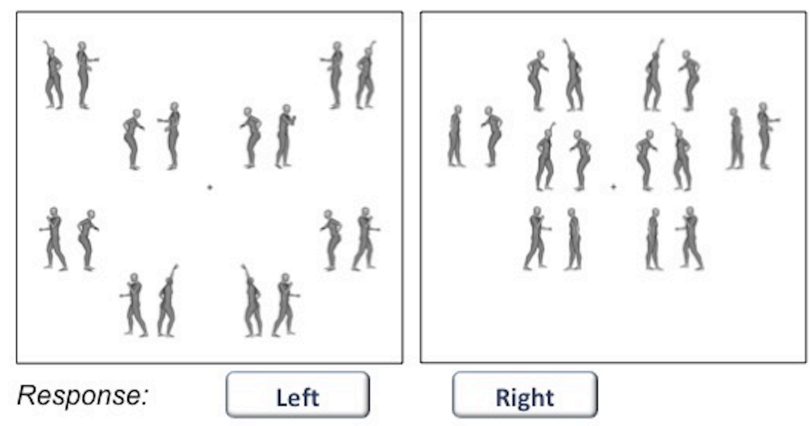

B
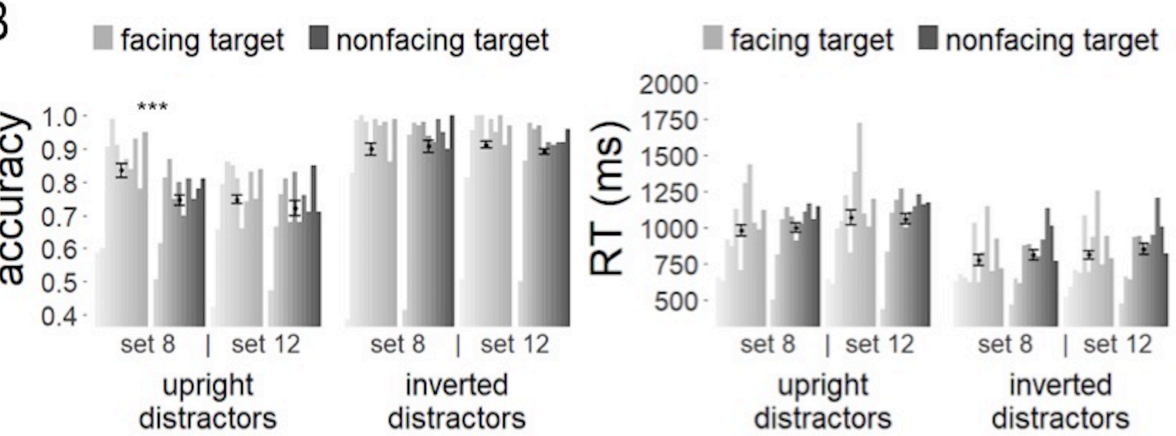

C facing target $\square$ nonfacing target
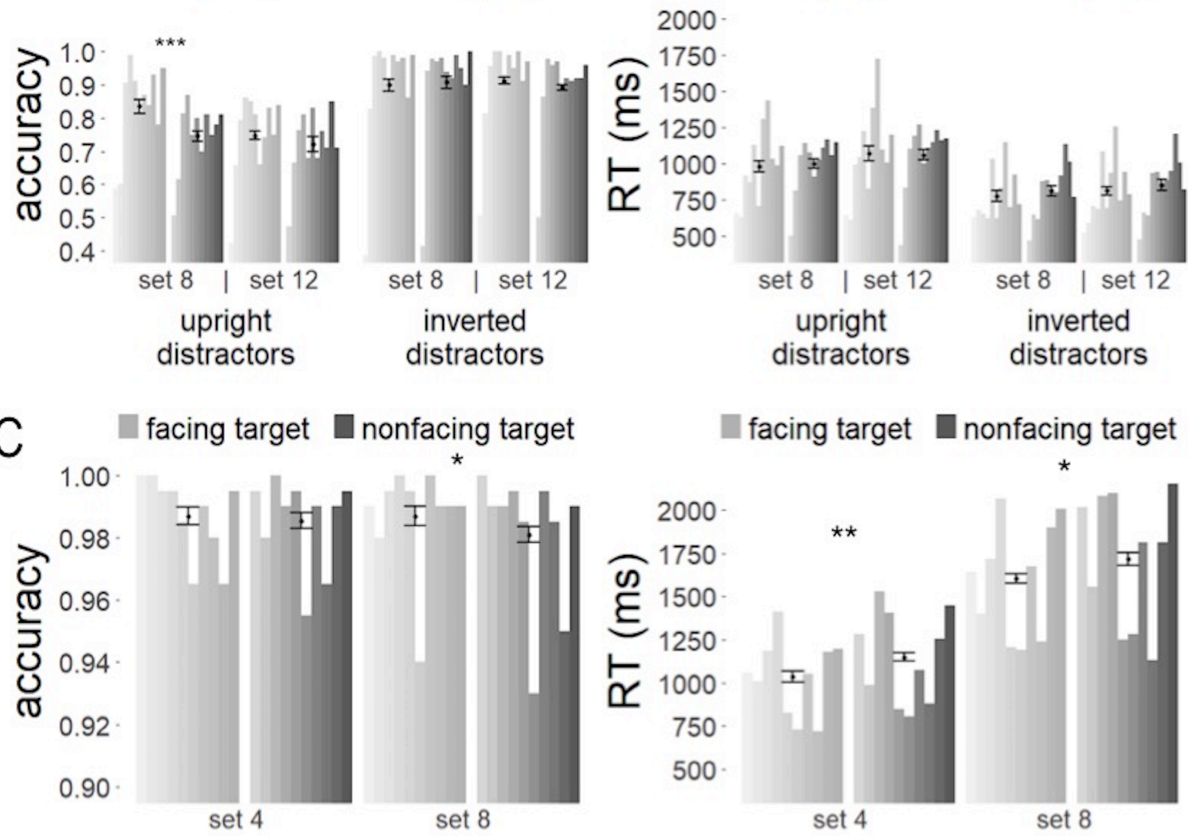

Figure 1. A) Examples of search arrays used as experimental stimuli and response keys as an illustration of the task in Experiments 1-2. B) Individual and group mean proportion of correct responses (accuracy) and RTs (ms) in Experiment 1 as function of target type (facing target or nonfacing target), set size (8 or 12) and distractors' orientation (uprightor inverted). C) Individual and group mean proportion of correct responses (accuracy) and RTs (in ms) in Experiment 2 as function of target type (facing target or nonfacing target) and set size (4 or 8). Each gray bar corresponds to one subject; the order of subjects is kept constant across conditions. Asterisks indicate the significant effect of target type, when found $\left(* * * p=.002,{ }^{* *} p=.01,{ }^{*} p\right.$ $=.05$ ). Error bars represent \pm 1 SEM within subjects (Cousineau, 2005). 


\section{Experiment 2 (39)}

Here, we focused on RTs and tested whether subjects detected facing dyads better than nonfacing dyads, when they had unlimited time to explore the whole array. We expected high accuracy and the asymmetry effect to shift to response latencies.

\section{Participants}

Ten healthy adults (7 female, 22 years $\pm 3.63 S D$ ) with normal or corrected-to-normal vision participated as paid volunteers, after signing an informed consent. Sensitivity power analysis (G*Power 3.1 ) showed that the current test of the interaction between target and set size (sample size $=10, \beta=0.80$, alpha $=0.05$ ) could detect a minimal effect size of $\eta_{p}{ }^{2}=0.318$.

\section{Stimuli and apparatus}

Using the same dyads of Experiment 1, search arrays of Experiment 2 had a set size of either four dyads (one target and three distractors, with two dyads at each side of the central fixation) or eight dyads (one target and seven distractors, with four dyads at each side of the central fixation). A total of 800 unique arrays were presented to each subject, including 400 with a facing target among nonfacing distractors (200 with set size of four and 200 with set size of eight) and 400 with a nonfacing target among facing distractors (200 with set size of four and 200 with set size of eight). The target was on the left side of the screen in half of the arrays, and on the right side, in the remaining arrays. Like in Experiment 1, the target always appeared at any of eight central locations. 


\section{Procedure}

Subjects were tested in the same setting of Experiment 1. In two separate blocks, they were instructed to search for the facing dyad among nonfacing dyads or vice versa. The order of blocks was counterbalanced across subjects. Task instructions were identical to Experiment 1: to report whether the target was on the left or right of central fixation, by pressing a key with the left and right index finger, respectively. In each trial, a central-fixation cross ( $200 \mathrm{~ms}$ ) was followed by a blank (700 ms), then, by the search array, which stayed on the screen until the subject responded. The time to respond was unlimited. The next trial began after $1400 \mathrm{~ms}$ from the response. Subjects could take a break every 40 trials, and in the interval between the two blocks. The experiment began with a familiarization block of 16 trials.

In summary, Experiment 2 differed from Experiment 1 for the following features. Arrays with set size of twelve were replaced by arrays with set size of four, because results of Experiment 1 had suggested that the former was too difficult to appreciate differences across conditions. Conditions with inverted distractors were discarded, as Experiment 1 had shown that search asymmetry only occurred when both target and distractors were upright. Moreover, closely matched facing and nonfacing dyads (see Stimuli and apparatus of Experiment 1) were used to create a unique set of 800 arrays for each subject. This made it very unlikely that systematic differences in visuo-spatial properties of the arrays -besides the relative positioning of bodies in targets and distractors (facing vs. nonfacing)- could bias the visual search performance. Finally, in Experiment 2, search arrays were presented for unlimited 
time, to induce an effect in RTs. Like in Experiment 1, the target was always in one of eight central locations.

\section{Results}

All subjects performed within 2.5 SD from the group mean in terms of accuracy (mean proportion of correct responses) and RTs, and were included in the analyses. A 2 target type (facing dyad, nonfacing dyad) $\times 2$ set size (four dyads, eight dyads) repeated-measures ANOVA on accuracy values yielded a main effect of target type, $F(1,9)=7.11, p=.026, \eta_{p}{ }^{2}=0.441$ (Figure 1c). This effect, reflecting higher accuracy in detecting the facing- target than for the nonfacing-dyad target, shall be taken with caution, since accuracy was overall high and variability, low. The set size and the interaction between the two factors yielded no significant effects, $F(1,9)<1$, ns.

RTs within $2 S D$ from the individual's mean and associated with correct responses (95\% of total values) were entered in a $2 \times 2$ ANOVA with factors: target type and set size. A main effect of target type showed an advantage of $~ 115 \mathrm{~ms}$ in searching for the facing dyad among nonfacing distractors, relative to the reverse condition, $F(1,9)$ $=8.39, p=0.018, \eta_{p}^{2}=0.482$. This effect was found in nine out of ten subjects. There was a significant effect of set size, $F(1,9)=186.69, p<0.001, \eta_{p}{ }^{2}=0.954$, but no interaction between the two, $F(1,9)<1$, ns (Figure 1c). As anticipated by the lack of target type by set size interaction, the search slope (time/item) was comparable in the two target-type conditions.

Generally, search was visibly less efficient (slower) in Experiment 2, relative to Experiment 1. In Experiment 2, participants had unlimited time to search, and they used that time. As a result, the effect in accuracy seen in Experiment 1 transferred to 
RTs in an analogous condition of Experiment 2 (set size of 8), and generalized to a new condition (set size of 4); in both experiments, the performance revealed an advantage in detecting facing-dyad, over nonfacing-dyad targets. Comparable search slopes for the two target-type conditions imply that search throughout the array (i.e., through facing versus nonfacing distractors) was equally efficient. Therefore, like in Experiment 1, the asymmetry is likely to reflect stronger recruitment of attention by the facing, than the nonfacing target.

\section{Experiment 3 (55)}

An asymmetry can occur because one type of distractors is easier to process than another. In this case, the asymmetry increases with the number of distractors to check and reject. Here, we set conditions to push the processing of distractors, and test whether search through facing distractors was more efficient than search through nonfacing distractors.

\section{Participants}

Eleven healthy adults (8 female, 22 years $\pm 4.69 S D$ ) with normal or corrected-tonormal vision participated as paid volunteers, after signing an informed consent. One subject was discarded because he was an outlier in terms of RTs (mean value >2.5 SD below the group mean). The minimal detectable effect size for the critical interaction in the current experiment was $\eta_{p}^{2}=0.203$ (sample size $=10, \beta=0.80$, alpha $=0.05)$.

\section{Stimuli and apparatus}


The same dyads of Experiment 1 (except the set with inverted distractors) were used in Experiment 3. Search arrays were structured as in Experiment 1: each half of the array included 16 cells. In $50 \%$ of arrays, one half was the mirrored version of the other. Thus, these arrays only included either nonfacing dyads (facing-target absent) or facing dyads (nonfacing-target absent). In the remaining $50 \%$ of trails, the two halves of the array differed for one cell, which displayed the target on one side and a distractor in the other side: when the target was a facing dyad all other items in the array were nonfacing dyads (facing-target present); when the target was a nonfacing dyad, all other items were facing dyads (nonfacing-target present). When present, the target could appear at any (central or more peripheral) location in the array. Set sizes varied from four to eight dyads. We matched the level of crowding across set sizes, by presenting always four dyads in each half of the array. Thereby, with a set size of four ( $50 \%$ of the trials), one half of the array was blank (the left one in $50 \%$ of trials, the right one in $50 \%$ of trials). For each subject, a unique set of arrays was created, 100 with one facing target among three nonfacing distractors, 100 with one nonfacing target among three facing distractors, 100 with four nonfacing distractors and 100 with four facing distractors. These conditions were replicated with a set size of eight dyads including either one target and seven distractors or eight distractors. In total, each subject saw 800 unique arrays.

\section{Procedure}

Subjects were tested in the same setting of Experiments 1-2. In Experiment 3, they were instructed to report whether the target was present or absent. They had to respond by pressing one of two keys of a computer keyboard with the left or right 
index finger, respectively (the mapping of a key -right/left arrow- to a response present/absence- was counterbalanced across subjects). The task included two blocks, one with the demand to search for the facing dyads, and the other, to search for the nonfacing dyad (the order of the blocks was alternated across subjects). Each trial began with a central-fixation cross $(200 \mathrm{~ms})$, followed by a blank screen (700 ms) and then by the search array, which stayed on the screen until the subject pressed a response-key. The next trial began after $1400 \mathrm{~ms}$ from the response. RTs and accuracy were recorded. Subjects could take a break every 40 trials, and in the interval between the two blocks. The experiment began with a familiarization block of 16 stimuli, two for each of the eight experimental conditions. The experiment lasted about 90 minutes.

In sum, to push the search throughout distractors, we introduced three changes in the paradigm, relative to Experiment 1. First, we increased target location uncertainty by having the target appearing at any location, so that it was not always available around central fixation. Second, the target was present only in $50 \%$ of trials (target present/absent task). In such task, target absence can be reported only after checking and rejecting every item in the array. To further promote this strategy, subjects were given unlimited time to search.

\section{Results}

Mean proportion of correct responses and mean RTs for every subject but one were within 2.5 SD from the group mean; therefore, the following analyses included a total of ten subjects. A 2 target type (facing dyad, nonfacing dyad) $\times 2$ set size (4 dyads, 8 dyads) $\times 2$ target presence (present, absent) repeated-measures ANOVA 
was conducted on accuracy values. The analysis yielded a main effect of set size, $F(1,9)=48.32, p<0.0001, \eta_{p}^{2}=0.843$, a main effect of target presence, $F(1,9)=$ 78.89, $p<0.0001, \eta_{p}^{2}=0.898$, and an interaction between the two, $F(1,9)=89.26, p$ $<.0001, \eta_{p}{ }^{2}=0.908$, reflecting a larger difference between the two set-size conditions in trials where the target was present, relative to those in which it was absent (Figure 2b). No other effect or interaction was significant (effect of target, $F(1,9)<1$, n.s; interactions between: target type and set size, $F(1,9)=1.58, p=0.240$; target type and target presence, $F(1,9)<1$, n.s.; target, set size and target presence, $F(1,9)=1.58, p=0.240)$.

RTs within $2 S D$ from the individual's mean and associated with a correct response (90\% of total values) were entered in a $2 \times 2 \times 2$ ANOVA with factors target type, set size and target presence. First, a main effect of target type showed a general advantage in search for the nonfacing target through facing distractors than for the facing target through nonfacing distractors, $F(1,9)=10.86, p=0.009, \eta_{p}{ }^{2}=.547$. Eight out of ten subjects showed this advantage. There was a significant effect of set size, $F(1,9)=275.43, p<0.001, \eta_{p}^{2}=0.968$, and, importantly, a significant interaction between target type and set size, $F(1,9)=13.26, p=0.005, \eta_{p}{ }^{2}=0.596$ (Figure 2b). In particular, subjects were faster in finding the nonfacing target among facing distractors than in the opposite condition, and this difference was more pronounced in trials with eight dyads, $t(9)=3.90, p=0.004$, than with four dyads, $t(9)=2.17, p=$ 0.058. Put in another way, RTs increased from the smaller to the larger set size, to a lesser extent when subjects searched thought facing distractors, then when they searched through nonfacing distractors. Therefore, the search slope was shallower (i.e., more efficient search) for the search through facing-dyad distractors than 
through nonfacing-dyad distractors. Also significant were the effect of target presence, $F(1,9)=239.90, p<0.0001, \eta_{p}{ }^{2}=.964$, and the interaction between set size and target presence, $F(1,9)=106.09, p<0.0001, \eta_{p}{ }^{2}=.922$. The last effect showed that the RT increase as a function of set size was more pronounced in the targetabsent (1406 ms on average), than in the target-present condition (760 ms on average). This is a well-known effect when using detection tasks (present/absent) in serial search (Treisman \& Gelade, 1980). The remaining effects were not significant (target type by target presence: $F(1,9)=3.87, p=0.081$; target type by set size by target presence: $F(1,9)=2.32, p=0.162$ ).

In sum, subjects were faster at finding the nonfacing than the facing target. Different search slope for the two target-type conditions demonstrates that the above asymmetry reflected more efficient search through facing distractors than through nonfacing distractors. Indeed, the search slope, estimating the time spent per item, primarily reflected the processing of distractors that constituted the majority of items in the array. Significantly different search slopes also when the target was absent confirmed that subjects' performance reflected how efficiently distractors were processed, aside from target detection. Thus, the task setting in Experiment 3 succeeded in driving search through the distractors. In this process, subjects were faster at checking and rejecting distractors when they were facing dyads than when they were nonfacing dyads. As a result of more efficient search through the facing distractors, nonfacing targets, when present, were found faster than facing targets. 
A

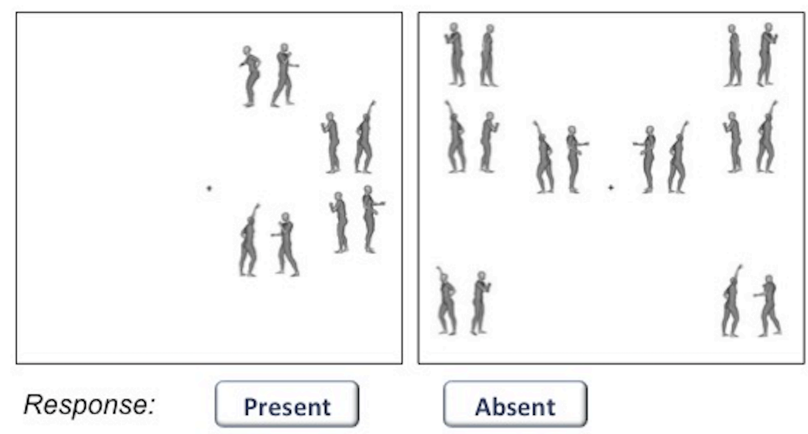

B

a facing target $\square$ nonfacing target

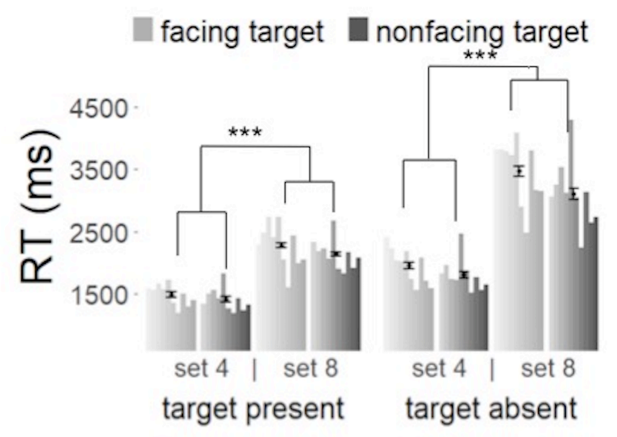

C

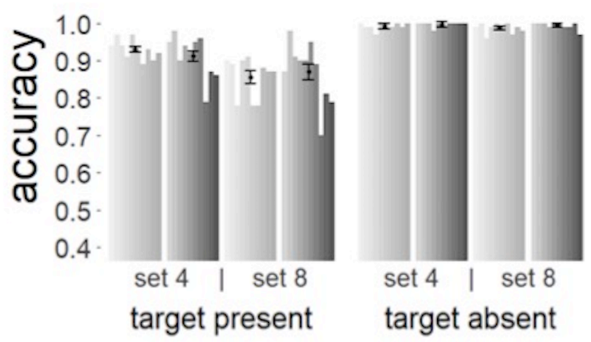

C a facing target $\square$ nonfacing target
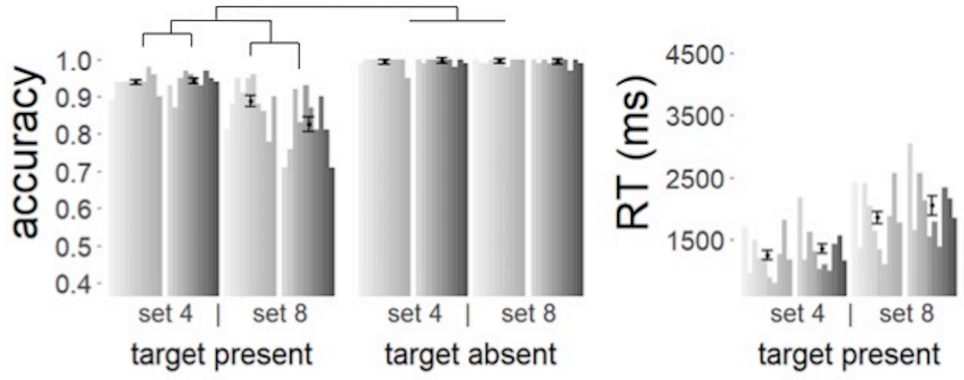

facing target $\square$ nonfacing target

Figure 2. A) Examples of search arrays used as experimental stimuli and response keys as an illustration of the task in Experiments 3-4. B) Individual (bars in gray) and group mean proportion of correct responses (accuracy) and RTs (ms) in Experiment 3 as function of target type (facing target or nonfacing target), set size (8 or 4 ) and target presence. Asterisks indicate significant target type by set size interaction in both target present and target absent conditions (*** $p=.005)$. B) Individual and group mean proportion of correct responses (accuracy) and RTs (ms) in Experiment 4 using the same design of Experiment 3. Asterisk indicates significant target type by set size interaction in target present, but not in target absent condition $\left({ }^{*} p=.025\right)$.

Error bars represent \pm 1 SEM within subjects (Cousineau, 2005). Each bar corresponds to one subject; the order of subjects is kept constant across conditions. 


\section{Experiment 4 (134)}

Experiments 1-2 showed asymmetric detection of facing versus nonfacing targets; Experiment 3 showed asymmetric processing of facing versus nonfacing distractors. We argue that the two outcomes are expressions of the same fundamental difference between processing facing versus nonfacing dyads, which manifests in a way or the other depending on task settings. On our view, by varying the target location uncertainty, we put more or less demand on processing of distractors: higher uncertainty pushed search through the distractors, yielding faster search for the nonfacing target (Experiment 3). To support this, in Experiment 4, everything was identical to Experiment 3, except for the target location, constrained to the central area, like in Experiments 1-2. With this change, we expected to restore the asymmetry in target detection seen in Experiments 1-2, using the task of Experiment 3.

\section{Participants}

Eleven healthy adults (7 female, 21 years $\pm 2.77 S D$ ) with normal or corrected-tonormal vision participated as paid volunteers, after signing an informed consent. One subject was discarded because he was an outlier in terms of RTs. Therefore, the minimal detectable effect size for the target type by set size by target presence interaction was $\eta_{p}{ }^{2}=0.203$ (sample size $=10, \beta=0.80$, alpha $=0.05$ ). 


\section{Stimuli, apparatus and procedure}

Everything was identical to Experiment 3, except for the target, which could appear only in one of the eight locations around central fixation. By minimizing the effect of eccentricity, the target was made readily available for detection.

\section{Results}

Mean proportion of correct responses and mean RTs were within 2.5 SD from the group mean, for every subject but one, which was excluded from the analysis.

A 2 target type (facing dyad, nonfacing dyad) $\times 2$ set size ( 4 dyads, 8 dyads) $\times 2$ target presence (present, absent) repeated-measures ANOVA was conducted on accuracy. The analysis showed an effect of target type, $F(1,9)=7.65, p=0.022, \eta_{p}^{2}=0.459$, reflecting higher accuracy in search for the facing, than for the nonfacing target. The analysis also showed a main effect of set size, $F(1,9)=26.37, p<0.001, \eta_{p}{ }^{2}=0.745$, and target presence, $F(1,9)=66.11, p<0.0001, \eta_{p}{ }^{2}=0.880$, and interactions between target type and set size, $F(1,9)=5.73, p=0.040, \eta_{p}{ }^{2}=0.389$, target type and target presence, $F(1,9)=5.29, p=0.047, \eta_{p}{ }^{2}=0.370$, and set size and target presence, $F(1,9)=23.14, p=0.001, \eta_{p}{ }^{2}=0.720$. Finally, the three way interaction was significant, $F(1,9)=7.27, p=0.025, \eta_{p}^{2}=0.447$ (Figure $2 c$ ). Pairwise comparisons showed that, analogously to Experiment 1, the advantage for the facing target among nonfacing distractors was significant in the condition with set size of eight and with the target present only, $t(9)=2.85, p=0.019$ (all other $p s>0.250$ ). Eight out of then subjects showed this advantage.

The same analysis was performed over mean RTs, considering values within $2 S D$ from the individual's mean and associated with a correct response $(90.45 \%$ of total 
values). Results show an effects of set size, $F(1,9)=136, p<0.0001, \eta_{p}{ }^{2}=0.938$, and of target presence, $F(1,9)=161.32, p<0.0001, \eta_{p}{ }^{2}=0.947$, and the interaction between the two, $F(1,9)=62.37, p<0.0001, \eta_{p}{ }^{2}=0.874$. No other effect was significant (target type: $F(1,9)<1$, n.s.; target type by set size: $F(1,9)<1$, n.s.; target type by target presence, $F(1,9)=2.58, p=0.143$; target type by set size by target presence, $F(1,9)=3.12, p=0.111$ ). As implied by the lack of interaction between target type and set size, the slope was comparable for the two target-type conditions.

The pattern of effects found in accuracy analysis showed that, when present and in predictable (central) locations - i.e., readily available for detection -, the facing target among nonfacing detractors was correctly recognized, more often than the nonfacing target among facing distractors. Hence, as expected, the asymmetry was expressed through a difference in detecting facing versus nonfacing targets. The lack of difference in search slope (i.e., interaction between target type and set size in RT) contributes to rule out an account of the performance based on different search efficiency through facing versus nonfacing distractors. In sum, Experiment 4 confirms that reducing target location uncertainty abolished performance differences that could be ascribed to processing of distractors.

\section{Experiment 5 (49)}

We tested whether grouping could mediate the search benefit for facing dyads, by measuring a putative cost in accessing local features of grouped dyadic 
representations. If facing bodies are perceptually grouped, search for one body should be harder when it is member of a facing versus a nonfacing dyad.

\section{Participants}

Eleven healthy adults (9 female, 20 years $\pm 3.20 S D$ ) with normal or corrected-tonormal vision participated as paid volunteers, after signing an informed consent. One subject was discarded due to significantly slower performance with respect to the group average. The current test had power to detect a minimal effect size of $\eta_{p}{ }^{2}$ $=0.199$ (sample size $=10, \beta=0.80$, alpha $=0.05)$ for the critical comparison between the two conditions (target in facing versus in nonfacing dyad).

\section{Stimuli and apparatus}

The search arrays of Experiment 5 were created analogously to the previous experiments, to include eight dyads, four on each side of the array. This time, every array included four facing and four nonfacing dyads. The target was one of two individuals: "punching body" (i.e., an individual in a punching pose), or "begging body" (i.e., an individual in a begging pose). While in an array, the same individual could appear several times, the target appeared only once, either in a facing or in a nonfacing dyad, on the left ( $50 \%$ of trials) or the right side of the array ( $50 \%$ of trials). The two halves of the array were separated by a central fixation cross. The dyad carrying the target could appear at one of the eight locations around central fixation. Each subject saw a total of 400 unique arrays with the target in either a facing (200) or a nonfacing dyad (200). 


\section{Procedure}

Subjects were tested in the same setting of Experiments 1-4. They performed one block with the two experimental conditions randomly interleaved. In each trial, after a central-fixation cross (200 ms), one of two words appeared for 1400 ms to cue the target (begging or punching). After a blank (700 ms), the search array appeared and stayed on the screen for 800 ms. Finally, a blank screen was shown until the subject provided a response. In each trial, subjects were instructed to search for either target, as cued by the word, and to press the left or right key (with the left and right index finger, respectively), to indicate the positioning of the target (left or right, relative to the central cross). Every 40 trials, subjects could take a break. Before starting the actual experiment, they were shown the images of the target-individuals for as much time as they needed, and completed a familiarization block of 16 trials.

\section{Results}

All subjects but one performed within $2.5 S D$ from the group mean in terms of accuracy and RTs. Therefore, the following analyses included ten subjects. We used a pairwise $t$-test to contrast the mean accuracy and RTs for conditions with target in a facing versus a nonfacing dyad. The target-individual was correctly detected more often when in a nonfacing, than in a facing dyad, $t(9)=3.41, p=0.008, \eta_{p}{ }^{2}=0.563$ (Figure 3). Nine out of ten subjects showed this effect. The RT analysis, including values within $2 S D$ from the individual's mean and associated with a correct response (71\% of total values), yielded no significant difference between the two conditions, $t(9)=1.13, p>.250$ (Figure 3). 
Here, analogously to Experiment 1, the target was presented at predictable (central) locations and the array was shown briefly. Because of the predictable target location and because, in this experiment, arrays always displayed the same number of facing and nonfacing distractors, the contribution of distractors processing to the effect was equal across the two conditions. Thus, the asymmetry in accuracy is attributed to processing of targets. In particular, we propose that individual (target) bodies are easier to detect in the nonfacing that in the facing dyad, because in the latter case pairs of bodies are perceptually grouped.

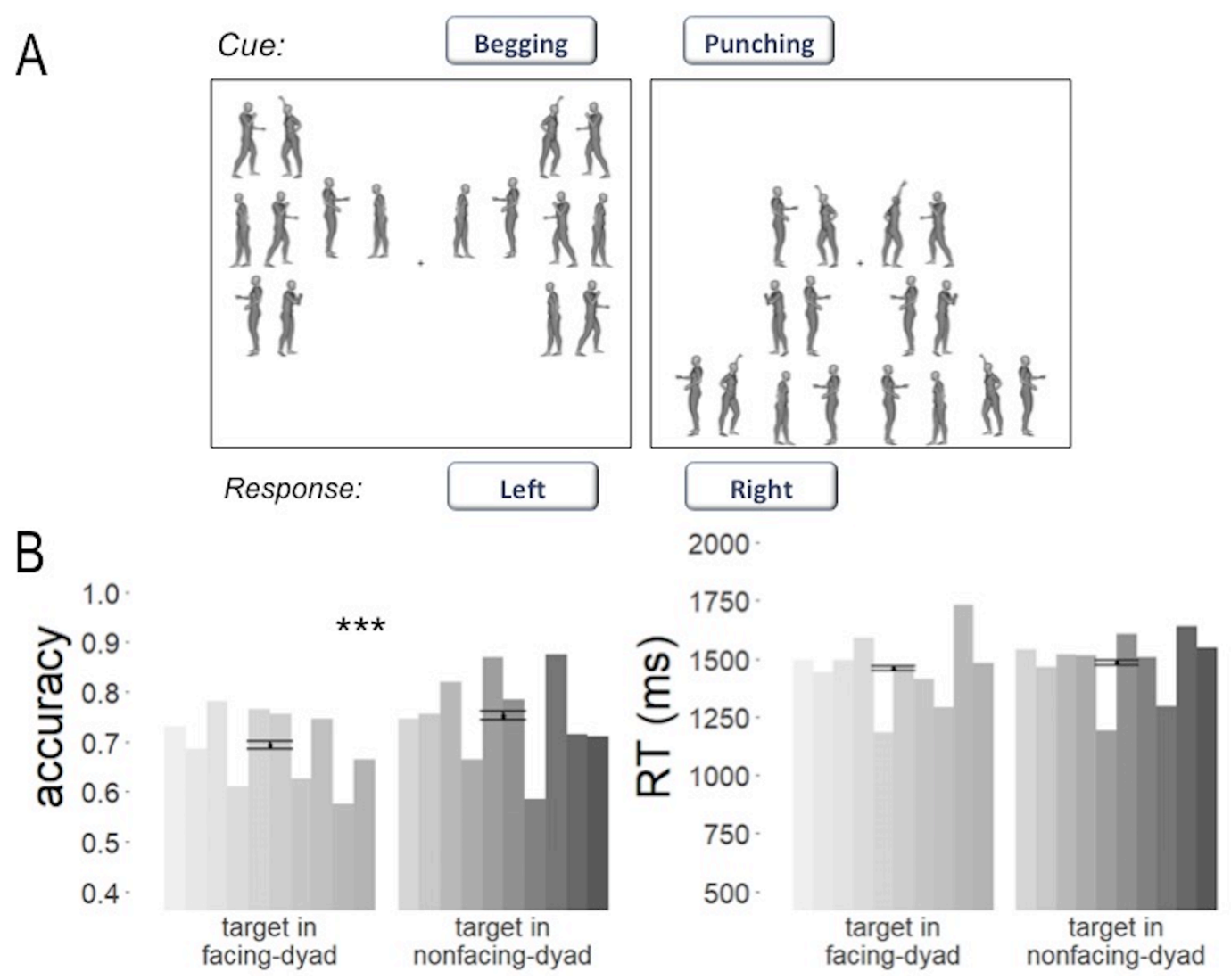

Figure 3. A) Examples of search arrays used as experimental stimuli and response keys as an illustration of the task in Experiment 5. B) Individual and group mean accuracy (proportion of correct responses) and RTs (ms) as function of whether the target was in a facing dyad (target in facing-dyad) or in a nonfacing dyad (target in nonfacing-dyad). Asterisks indicate a significant difference $(* * * p=.008)$. Error bars represent \pm 1 SEM within subjects (Cousineau, 2005). Each gray bar corresponds to one subject; the order of subjects is kept constant across conditions. 


\section{Discussion (798 words)}

In cluttered environments, humans show priority and particularly efficient detection and recognition of socially relevant entities such as faces and bodies. But, how do humans parse a crowd, where socially relevant stimuli are everywhere?

Experiments 1-4 demonstrate that during visual search there is rapid access to relations between multiple bodies, with particularly efficient processing of facing seemingly interacting- body dyads. Experiment 5 indicates that perceptual grouping may be a likely candidate as the mechanism underlying efficient processing of facing dyads.

The occurrence of search asymmetry between facing and nonfacing dyads suggests that the relative positioning of bodies accounts for how individuals parse people in visual environments. A search asymmetry can occur because a stimulus type carries a property that is salient/easier to detect, and is absent in another stimulus (Treisman \& Souther, 1985). In Experiment 1, we asked whether, in a crowd, facing dyads are more likely to recruit attention than nonfacing dyads. In this test, the target could appear only in a subset of central locations, for a short time, to minimize eye movements and covert shifts of attention away from central area, and favor detection (Carrasco et al., 1998; Wolfe et al., 2011). We found that subjects detected correctly facing targets more often than nonfacing targets. Given comparable search slopes across conditions, we conclude that the asymmetry reflected how strongly the target could recruit attention, rather than how efficiently the distractors were processed. Experiment 2, where arrays were presented for unlimited time, replicated this pattern in RTs and, to a lesser extent, in accuracy. 
Experiment 3 was designed to exploit another source of asymmetry: the speed at which distractors are checked and rejected. Different search slopes depending on the type of distractors showed that the new task successfully promoted processing of distractors. As a result, the pattern of Experiment 3 was the opposite of Experiments 1-2, with faster search for nonfacing targets amongst facing distractors, than for facing targets amongst nonfacing distractors. In Experiment 4, we replicated the conditions of Experiment 3 but with target appearance constrained to central locations, as in Experiments 1-2. This change was sufficient to restore, in a different task, the effect seen in Experiments 1-2: more predictable (and central) target location favored target detection, which emphasized a stronger recruitment of attention by facing than nonfacing targets.

While we do not know which specific feature of facing dyads accounts for the asymmetry, our results encourage the thinking that facing dyads fall in the same biologically-relevant category as faces or bodies, associated with high visual sensitivity, rapid discrimination and spontaneous recruitment of attention (Birmingham et al., 2008; Downing et al., 2004; Gobbini, Gors, Halchenko, Hughes, \& Cipolli; New et al., 2007; Langton, Law, Burton, \& Schweinberger, 2008). In line with this view, interacting bodies are recognized faster and better than unrelated bodies in low-visibility conditions (Papeo, Stein, \& Soto-Faraco, 2017; see also Vestner, Tipper, Hartley, Over, \& Rueschemeyer, 2019). Moreover, just like for faces and bodies, perception of interacting dyads has been associated with a behavioral signature of high visual sensitivity (i.e., large cost of inversion; Papeo \& Abassi, 2019), and with neural selectivity in posterior temporal regions (Isik, Koldewyn, Beeler, \& Kanwisher, 2017; Walbrin, Downing, \& Koldewyn, 2018). 
High visual sensitivity to a stimulus indexed by behavioral and neural effects, predicts an attentional/perceptual advantage. The current study substantiates that prediction: whether they are targets to detect, or distractors to check-and-reject, facing dyads are processed more efficiently than nonfacing dyads. A mechanism that could account for particularly efficient processing of multi-part objects is perceptual grouping, the automatic combination of parts into a whole (Green \& Hummel, 2006; McMains \& Kastner, 2010). In Experiment 5, we exposed this mechanism through one of its diagnostic features, the cost of individuating components of the group, indicated by poorer access to single elements (i.e., bodies) within the dyad.

An analogous phenomenon has previously been reported for faces: efficient search for a face is contrasted by inefficient search for one part of that whole (e.g., the mouth) (Suzuki \& Cavanagh, 1995). Those findings demonstrated that visual search gives priority to more a global level of representation over a more local one.

In conclusion, various lines of research have demonstrated the attentional/perceptual benefit of faces and bodies when they appear amongst other entities. The present study describes a mechanism to deal with real-world crowed environments, where faces and bodies are everywhere. Human attention has rapid access to people as well as relations among people, and can use those relations to parse crowded scenarios. In this process, high priority is given to seemingly interacting bodies over non-interacting bodies. The former trigger a stronger recruitment of attention and appear to be perceptually grouped as a unit, with concurrent benefits (efficient processing of the whole) and costs (poor access to local features). 


\section{Author contribution}

L. Papeo and S. Soto-Faraco developed the study concept. Study design, data collection, and analyses were performed by L. Papeo and N. Goupil. L. Papeo drafted the manuscript, and S. Soto-Faraco provided critical revisions. All authors approved the final version of the manuscript for submission. 


\section{Acknowledgements (25)}

The authors thank Leonor Castro and Lisa Boinon for help with data collection and Jean-Remy Hochmann for comments on an earlier version of the manuscript. 


\section{References}

Birmingham, E., \& Kingstone, A. (2009). Human social attention. Annals of the New York Academy of Sciences, 1156(1), 118-140.

Brainard, D. H. (1997). The Psychophysics Toolbox. Spatial Vision, 10, 433-436.

Cousineau, D. (2005). Confidence intervals in within-subject designs: A simpler solution to Loftus and Masson's method. Tutorials in Quantitative Methods for Psychology, 1, 42-45

Downing, P. E., Bray, D., Rogers, J., \& Childs, C. (2004). Bodies capture attention when nothing is expected. Cognition, 93, B27-B38.

Duncan, J., \& Humphreys, G. W. (1989). Visual search and stimulus similarity. Psychological review, 96(3), 433.

Gobbini, M. I., Gors, J. D., Halchenko, Y. O., Hughes, H. C., \& Cipolli, C. (2013). Processing of invisible social cues. Consciousness and Cognition, 22, 765-770.

Green, C., \& Hummel, J. E. (2006). Familiar interacting object pairs are perceptually grouped. Journal of Experimental Psychology: Human Perception and Performance, $32,1107-1119$.

Isik, L., Koldewyn, K., Beeler, D., \& Kanwisher, N. (2017). Perceiving social interactions in the posterior superior temporal sulcus. Proceedings of the National Academy of Sciences, 201714471.

Kaiser, D., Stein, T., \& Peelen, M. V. (2014). Object grouping based on real-world regularities facilitates perception by reducing competitive interactions in visual cortex. Proceedings of the National Academy of Sciences, USA, 111, 11217-11222. 
Kwak, H. W., Dagenbach, D., \& Egeth, H. (1991). Further evidence for a timeindependent shift of the focus of attention. Perception \& Psychophysics, 49(5), 473480.

Langton, S. R. H., Law, A. S., Burton, A. M., \& Schweinberger, S. R. (2008). Attention capture by faces. Cognition, 107, 330-342.

McMains, S.A., \& Kastner, S. (2010). Defining the units of competition: Influences of perceptual organization on competitive interactions in human visual cortex. Journal of Cognitive Neuroscience, 22, 2417-2426.

New, J., Cosmides, L., \& Tooby, J. (2007). Category-specific attention to animals reflects ancestral priorities, not expertise. Proceedings of the National Academy of Sciences of the United States of America, 104, 16598-16603.

Papeo, L., Stein, T., \& Soto-Faraco, S. (2017). The two-body inversion effect. Psychological science, 28(3), 369-379.

Papeo, L., Wurm, M. F., Oosterhof, N. N., \& Caramazza, A. (2017). The neural representation of human versus nonhuman bipeds and quadrupeds. Scientific reports, $7(1), 14040$.

Powell, L. J., \& Spelke, E. S. (2018). Human infants' understanding of social imitation: Inferences of affiliation from third party observations. Cognition, 170, 31-48.

Quadflieg, S., \& Koldewyn, K. (2017). The neuroscience of people watching: how the human brain makes sense of other people's encounters. Annals of the New York Academy of Sciences, 1396(1), 166-182. 
Reed, C. L., Stone, V. E., Grubb, J. D., \& McGoldrick, J. E. (2006). Turning configural processing upside down: Part and whole body postures. Journal of Experimental Psychology: Human Perception and Performance, 32, 73-87.

Riddoch, M. J., Humphreys, G. W., Edwards, S., Baker, T., \& Willson, K. (2003). Seeing the action: Neuropsychological evidence for action-based effects on object selection. Nature Neuroscience, 6, 82-89.

Suzuki, S., \& Cavanagh, P. (1995). Facial organization blocks access to low-level features: An object inferiority effect. Journal of Experimental Psychology: Human Perception and Performance, 21(4), 901.

Treisman, A. M., \& Gelade, G. (1980). A feature-integration theory of attention. Cognitive psychology, 12(1), 97-136.

Treisman, A., \& Souther, J. (1985). Search asymmetry: A diagnostic for preattentive processing of separable features. Journal of Experimental Psychology: General, 114(3), 285.

Vestner, T., Tipper, S. P., Hartley, T., Over, H., \& Rueschemeyer, S. A. (2019). Bound together: Social binding leads to faster processing, spatial distortion, and enhanced memory of interacting partners. Journal of Experimental Psychology: General.

Walbrin, J., Downing, P., \& Koldewyn, K. (2018). Neural responses to visually observed social interactions. Neuropsychologia, 112, 31-39.

Wolfe, J. M. (2001). Asymmetries in visual search: An introduction. Perception \& psychophysics, 63(3), 381-389. 
Table 1. Summary of task settings in Experiments (Exp.) 1-5

\begin{tabular}{|c|c|c|c|c|c|c|}
\hline & \multicolumn{2}{|c|}{ Target location } & \multicolumn{2}{|c|}{ Stimulus duration } & \multicolumn{2}{|c|}{ Task } \\
\hline & Central & Everywhere & $\begin{array}{c}\text { Short } \\
\text { (800ms) }\end{array}$ & $\begin{array}{l}\text { Until } \\
\text { response }\end{array}$ & $\begin{array}{l}\text { Side } \\
(L / R)\end{array}$ & $\begin{array}{c}\text { Presence } \\
\text { (P/A) }\end{array}$ \\
\hline Exp. 1 & $\bullet$ & & $\bullet$ & & $\bullet$ & \\
\hline Exp. 2 & $\bullet$ & & & $\bullet$ & $\bullet$ & \\
\hline Exp. 3 & & $\bullet$ & & $\bullet$ & & $\bullet$ \\
\hline Exp. 4 & $\bullet$ & & & $\bullet$ & & $\bullet$ \\
\hline Exp. 5 & • & & • & & • & \\
\hline
\end{tabular}

Notes. Target location: central = in one of eight locations around central fixation; everywhere $=$ in central or more peripheral locations; Experimental task: side $(R / L)=$ to indicate whether the target was in the left $(L)$ of right $(R)$ side of the array; Presence $(P / A)=$ to indicate whether the target was present $(P)$ or absent $(A)$. 